EESTI NSV TEADUSTE AKADEEMIA TOIMETISED. XII KÖIDE FOUSIKA-MATEMAATIKA- JA TEHNIKATEADUSTE SEERIA. 1963, NR. 2

ИЗВЕСТИЯ АҚАДЕМИИ НАУК ЭСТОНСКОИ ССР. ТОМ ХІІ СЕРИЯ ФИЗИКО-МАТЕМАТИЧЕСКИХ И ТЕХНИЧЕСКИХ НАУК. 1963, № 2

\title{
ОБ ОДНОМ КЛАССЕ ИТЕРАЦИОННЫХ МЕТОДОВ В ПРОСТРАНСТВЕ ГИЛЬБЕРТА
}

\author{
С. Ульм, \\ кандидат физико-математических наук
}

1. А. С. Сергеев $\left.{ }^{5}\right]$ исследовал сходимость метода хорд для нелинейного операторного уравнения

$$
P(x)=0
$$

в пространстве Банаха. Автором [6] были предложены некоторые итерационные методы интерполяционного типа, содержащие аналог разделенных разностей второго порядка оператора $P(x)$. Для изучения сходимости этих методов был применен принцип мажорант.

Для приближенного решения уравнения (1) в вещественном пространстве Гильберта $H$ существует ряд методов [4] типа

$$
x_{n+1}=x_{n}-\varepsilon_{n}\left[P^{\prime}\left(x_{n}\right)\right]^{*} P\left(x_{n}\right) \quad n=0,1, \ldots,
$$

например, итерационные методы с минимальными невязками [3,2] и ошибками $\left[{ }^{7}\right]^{\star}$.

По сравнению с методом Ньютона эти методы обладают малой скоростью сходимости, но практическое применение их значительно проще, так как при методе Ньютона на каждом шаге требуется нахождение решения линейного уравнения.

Так как часто оказывается трудным и вычисление производной оператора $P(x)$, то при практических вычислениях представляют интерес методы класса

$$
x_{n+1}=x_{n}-\varepsilon_{n} P *\left(x_{n}, x_{n-1}\right) P\left(x_{n}\right) \quad n=0,1, \ldots,
$$

исследование которых и является целью нашей статьи.

Здесь $\varepsilon_{n}$ - положительные числа, причем $\underset{n}{\inf } \varepsilon_{n}>0 ; P\left(x^{\prime}, x^{\prime \prime}\right)$ аналог разделенных разностей первого порядка [5] для оператора $P(x)$; $P^{*}\left(x^{\prime}, x^{\prime \prime}\right)$ - оператор, сопряженный к линейному оператору $P\left(x^{\prime}, x^{\prime \prime}\right)$. От оператора $P\left(x^{\prime}, x^{\prime \prime}\right)$ мы требуем симметрии, т. е. $P\left(x^{\prime}, x^{\prime \prime}\right)=$ $=P\left(x^{\prime \prime}, x^{\prime}\right)$; если же $x^{\prime}=x^{\prime \prime}=x$, то $P(x, x)=P^{\prime}(x)$, где $P^{\prime}(x)$ - про-

\footnotetext{
* Методы класса $x_{n+1}=x_{n}-\varepsilon_{n} P\left(x_{n}\right)$ были рассмотрены М. М. Вайнбергом ['].
} 
изводная оператора $P(x)$ в смысле Гато. Основным для $P\left(x^{\prime}, x^{\prime \prime}\right)$ является соотношение

$$
P\left(x^{\prime}, x^{\prime \prime}\right)\left(x^{\prime}-x^{\prime \prime}\right)=P\left(x^{\prime}\right)-P\left(x^{\prime \prime}\right) .
$$

Предположим еще, что $P(x)$ является непрерывным в интересующей нас области.

\section{2. Сходимость методов класса (3) характеризует}

Те орем а 1. Пусть выполнены условия:

$1^{\circ} \quad\left\|P\left(x_{0}\right)\right\| \leqslant \eta_{0} ; \quad\left\|x_{0}-x_{-1}\right\| \leqslant \gamma ; \quad x_{0} \neq x_{-1}$;

$2^{\circ}$ длякаждого $x^{\prime}, x^{\prime \prime}, x^{\prime \prime \prime}$ из замкнутой сферь

$$
S\left[x_{0} ; \max \left(\gamma ; \frac{\varepsilon V \bar{K} \eta_{0}}{1-q}\right)\right]
$$

u $h \in H$ справедливы оценки:

$$
\begin{aligned}
& \text { a) }\left(P\left(x^{\prime}, x^{\prime \prime}\right) P^{*}\left(x^{\prime \prime}, x^{\prime \prime \prime}\right) h, h\right) \geqslant \frac{1}{M}\|h\|^{2} \quad 0<M<+\infty \\
& \text { б) }\left\|P\left(x^{\prime}, x^{\prime \prime}\right)\right\| \leqslant \sqrt{\bar{K}} \\
& 3^{\circ} \quad \varepsilon=\sup _{n} \varepsilon_{n}<\frac{2}{M K^{2}} .
\end{aligned}
$$

Тогда уравнение (1) имеет в сфере (5) единственное решение $x^{*}$, к которому последовательность (3) сходится со скоростью

где

$$
\left\|x^{*}-x_{n}\right\| \leqslant \frac{\varepsilon \sqrt{K} \eta_{0}}{1-q} q^{n} \quad n=0,1, \ldots
$$

$$
q=\sup _{n} \sqrt{K^{2} \varepsilon_{n}^{2}-\frac{2}{M} \varepsilon_{n}+1}
$$

Доказ ательств о: Исходя из соотношения (4), найдем:

$$
\begin{aligned}
\left\|P\left(x_{n+1}\right)\right\|^{2} & =\left\|P\left(x_{n}\right)+P\left(x_{n+1}, x_{n}\right)\left(x_{n+1}-x_{n}\right)\right\|^{2}= \\
& =\left\|P\left(x_{n}\right)-\varepsilon_{n} P\left(x_{n+1}, x_{n}\right) P^{*}\left(x_{n}, x_{n-1}\right) P\left(x_{n}\right)\right\|^{2}= \\
& =\left\|P\left(x_{n}\right)\right\|^{2}-2 \varepsilon_{n}\left(P\left(x_{n+1}, x_{n}\right) P^{*}\left(x_{n}, x_{n-1}\right) P\left(x_{n}\right), P\left(x_{n}\right)\right)+ \\
& +\varepsilon_{n}^{2}\left\|P\left(x_{n+1}, x_{n}\right) P^{*}\left(x_{n}, x_{n-1}\right) P\left(x_{n}\right)\right\|^{2} \leqslant \\
& \leqslant\left(K^{2} \varepsilon_{n}{ }^{2}-\frac{2 \varepsilon_{n}}{M_{i}}+1\right)\left\|P\left(x_{n}\right)\right\|^{2} \leqslant q^{2}\left\|P\left(x_{n}\right)\right\|^{2}<\left\|P\left(x_{n}\right)\right\|^{2} .
\end{aligned}
$$

Поскольку

TO

$$
\left\|x_{n+1}-x_{n}\right\| \leqslant \varepsilon_{n} V^{\prime} \bar{K}\left\|P\left(x_{n}\right)\right\| \quad n=0,1, \ldots,
$$

$$
\begin{gathered}
\left\|x_{n+p}-x_{n}\right\| \leqslant\left\|x_{n+p}-x_{n+p-1}\right\|+\ldots+\left\|x_{n+1}-x_{n}\right\| \leqslant \\
\leqslant \varepsilon \sqrt{K}\left(\left\|P\left(x_{n+p-1}\right)\right\|+\ldots+\left\|P\left(x_{n}\right)\right\|\right) .
\end{gathered}
$$


Обозначим $\eta_{R}=\left\|P\left(x_{k}\right)\right\|$. Тогда

H

$$
\eta_{k} \leqslant q^{k} \eta_{0} \quad k=1,2, \ldots,
$$

$$
\begin{gathered}
\left\|x_{n+p}-x_{n}\right\| \leqslant \varepsilon \sqrt{K}\left(q^{n+p-1} \eta_{0}+\ldots+q^{n} \eta_{0}\right)= \\
=\varepsilon \sqrt{K} \eta_{0}\left(1+q+\ldots+q^{p-1}\right) q^{n}
\end{gathered}
$$

Переходя к пределу $(p \rightarrow \infty)$, получим (6). Из непрерывности оператора $P(x)$ следует, что

$$
\left\|P\left(x^{*}\right)\right\|=\lim _{n \rightarrow \infty}\left\|P\left(x_{n}\right)\right\| \leqslant\left\|P\left(x_{0}\right)\right\| \cdot \lim _{n \rightarrow \infty} q^{n}=0
$$

т. е. $x^{*}$ является решением уравнения (1).

Легко увидеть, что элементы $x_{-1}, x_{0}, \ldots, x_{n}, \ldots, x^{*}$ принадлежат сфере (5).

Единственность решения в сфере Взяв в этом условии $x^{\prime \prime \prime}=x^{\prime}$, получим

(5) вытекает из условия $2^{\circ} \mathrm{a}$.

$$
\left\|P^{*}\left(x^{\prime}, x^{\prime \prime}\right) h\right\| \geqslant \frac{1}{\sqrt{M}}\|h\|,
$$

откуда вытекает существование левой обратной $\left[P^{*}\left(x^{\prime}, x^{\prime \prime}\right)\right]^{-1}$ и оценка $\left\|\left[P^{*}\left(x^{\prime}, x^{\prime \prime}\right)\right]^{-1}\right\| \leqslant \sqrt{M}$. Допустим, что в сфере (5) имеются два различных решения уравнения (1) $x^{*}$ и $x^{* *}$. Взяв в условии $2^{\circ}$ а $x^{\prime}=x^{\prime \prime \prime}=$ $=x^{*}, x^{\prime \prime}=x^{* *}$ и $h=\left[P^{*}\left(x^{*}, x^{* *}\right)\right]^{-1}\left(x^{*}-x^{* *}\right)$, получим

$$
0=\left(P\left(x^{*}, x^{* *}\right) P^{*}\left(x^{*}, x^{* *}\right)\left[P^{*}\left(x^{*}, x^{* *}\right)\right]^{-1}\left(x^{*}-x^{* *}\right),\right.
$$

$\left.\left[P^{*}\left(x^{*}, x^{* *}\right)\right]^{-1}\left(x^{*}-x^{* *}\right)\right) \geqslant \frac{1}{M}\left\|\left[P^{*}\left(x^{*}, x^{* *}\right)\right]^{-1}\left(x^{*}-x^{* *}\right)\right\|^{2}$,

откуда $\left[P^{*}\left(x^{*}, x^{* *}\right)\right]^{-1}\left(x^{*}-x^{* *}\right)=0$ или $x^{*}=x^{* *}$.

Теорема доказана.

3 амечание 1: Если при вычислениях на некотором шаге $x_{n+1}=x_{n}$, то $x_{n}=x^{*}$. Действительно, в том случае $P^{*}\left(x_{n}, x_{n-1}\right) P\left(x_{n}\right)=0$, а так как существует левая обратная $\left[P^{*}\left(x_{n}, x_{n-1}\right)\right]^{-1}$, то $P\left(x_{n}\right)=0$, T. e. $x_{n}=x^{*}$. щая

3. Пусть известна оценка $\left\|x^{*}-x_{0}\right\| \leqslant r$. Тогда справедлива следую-

Т ео рем а 2: Пусть выполнены условия:

$1^{\circ}\left\|x_{0}-x_{-1}\right\| \leqslant \gamma ; x_{0} \neq x_{-1}$;

$2^{\circ}$ для каждого $x^{\prime}, x^{\prime \prime}, x^{\prime \prime \prime} \in S\left[x_{0} ; \max (\gamma ;(1+q) r)\right]$ справедливы оценки:

a) $\left(P^{*}\left(x^{\prime}, x^{\prime \prime}\right) P\left(x^{\prime}, x^{\prime \prime \prime}\right)\left(x^{\prime}-x^{\prime \prime \prime}\right), x^{\prime}-x^{\prime \prime \prime}\right) \geqslant \frac{1}{M}\left\|x^{\prime}-x^{\prime \prime \prime}\right\|^{2}$, $0<M<+\infty$; $^{\star}$

- Условие $2^{\circ}$ а равносильно условию

$\left(P\left(x^{\prime}, x^{\prime \prime}\right)\left(x^{\prime}-x^{\prime \prime \prime}\right), P\left(x^{\prime}\right)-P\left(x^{\prime \prime \prime}\right)\right) \geqslant \frac{1}{M}\left\|x^{\prime}-x^{\prime \prime \prime}\right\|^{2}$. 
б) $\left\|P^{*}\left(x^{\prime}, x^{\prime \prime}\right) P\left(x^{\prime}, x^{\prime \prime \prime}\right)\right\| \leqslant K$;

$3^{\circ} \varepsilon=\sup _{n} \varepsilon_{n}<\frac{2}{M K^{2}}$.

Тогда последовательность (3) сходится к единственному в сфере (15) решению уравнения (1), причем

2วe

$$
\left\|x^{*}-x_{n}\right\| \leqslant q^{n} r \quad n=0,1, \ldots
$$

$$
q=\sup _{n} \sqrt{K^{2} \varepsilon_{n}^{2}-\frac{2}{M} \varepsilon_{n}+1}
$$

Д о к а з а тельст в о: Используя равенство

$$
P\left(x_{n}\right)=P\left(x^{*}, x_{n}\right)\left(x_{n}-x^{*}\right)
$$

и условия теоремы, получим:

$$
\begin{aligned}
\left\|x_{n+1}-x^{*}\right\|^{2}= & \left\|x_{n}-x^{*}-\varepsilon_{n} P^{*}\left(x_{n}, x_{n-1}\right) P\left(x_{n}\right)\right\|^{2}= \\
= & \left\|x_{n}-x^{*}\right\|^{2}-2 \varepsilon_{n}\left(x_{n}-x^{*}, P^{*}\left(x_{n}, x_{n-1}\right) P\left(x_{n}\right)\right)+ \\
& +\varepsilon_{n}^{2}\left\|P^{*}\left(x_{n}, x_{n-1}\right) P\left(x_{n}\right)\right\|^{2}= \\
= & \left\|x_{n}-x^{*}\right\|^{2}-2 \varepsilon_{n}\left(P^{*}\left(x_{n}, x_{n-1}\right) P\left(x^{*}, x_{n}\right)\left(x_{n}-x^{*}\right), x_{n}-x^{*}\right)+ \\
& +\varepsilon_{n}^{2}\left\|P^{*}\left(x_{n}, x_{n-1}\right) P\left(x^{*}, x_{n}\right)\left(x_{n}-x^{*}\right)\right\|^{2} \leqslant \\
& \leqslant\left\|x_{n}-x^{*}\right\|^{2}-\frac{2}{M} \varepsilon_{n}\left\|x_{n}-x^{*}\right\|^{2}+\varepsilon_{n}^{2} K^{2}\left\|x_{n}-x^{*}\right\|^{2}= \\
=\left(1-\frac{2}{M} \varepsilon_{n}\right. & \left.+K^{2} \varepsilon_{n}^{2}\right)\left\|x_{n}-x^{*}\right\|^{2} \leqslant q^{2}\left\|x_{n}-x^{*}\right\|^{2}<\left\|x_{n}-x^{*}\right\|^{2} .
\end{aligned}
$$

Отсюда легко получить оценку (16). Так как

$$
\left\|x_{-1}-x_{0}\right\| \leqslant \gamma
$$

$\left\|x_{k}-x_{0}\right\| \leqslant\left\|x_{k}-x^{*}\right\|+\left\|x^{*}-x_{0}\right\| \leqslant\left(1+q^{k}\right) r \leqslant(1+q) r$,

$$
k=1,2, \ldots \text {, }
$$

то элементы $x_{-1}, x_{0}, \ldots, x_{n}, \ldots, x^{*}$ принадлежат сфере (15). Единственность решения вытекает из условия $2^{\circ}$ a. Допустим, что в сфере (15) имеются два различных решения $x^{*}$ и $x^{* *}$. Взяв в условии $2^{\circ}$ а $x^{\prime}=x^{*}$, $x^{\prime \prime}=x^{\prime \prime \prime}=x^{* *}, \quad$ получим: $0=\left(P^{*}\left(x^{*}, x^{* *}\right) P\left(x^{*}, x^{* *}\right)\left(x^{*}-x^{* *}\right), \quad x^{*}-\right.$ $\left.-x^{* *}\right) \geqslant \frac{1}{M}\left\|x^{*}-x^{* *}\right\|^{2}$, откуда $x^{*}=x^{* *}$.

Теорема доказана.

4. Рассмотрим интерполяционный аналог итерационного метода с минимальными невязками

$$
x_{n+1}=x_{n}-\frac{\left\|P^{*}\left(x_{n}, x_{n-1}\right) P\left(x_{n}\right)\right\|^{2}}{\left\|P\left(x_{n}, x_{n-1}\right) P^{*}\left(x_{n}, x_{n-1}\right) P\left(x_{n}\right)\right\|^{2}} P^{*}\left(x_{n}, x_{n-1}\right) P\left(x_{n}\right) .
$$

В данном случае

$$
\varepsilon_{n}=\frac{\left\|P^{*}\left(x_{n}, x_{n-1}\right) P\left(x_{n}\right)\right\|^{2}}{\left\|P\left(x_{n}, x_{n-1}\right) P^{*}\left(x_{n}, x_{n-1}\right) P\left(x_{n}\right)\right\|^{2}},
$$

и имеет место 
Т ео рем а 3: Пусть выполнены условия:

$1^{\circ} \quad\left\|P\left(x_{0}\right)\right\| \leqslant \eta_{0} ; \quad\left\|x_{0}-x_{-1}\right\| \leqslant \gamma ; \quad x_{0} \neq x_{-1} ;$

$2^{\circ}$ длякањдого $x^{\prime}, x^{\prime \prime}, x^{\prime \prime \prime} \in S\left[x_{0} ; \max \left(\gamma ; \frac{\sqrt{M} \eta_{0}}{1-q}\right)\right]$

u $h \in H$ справедливы оценки:

a) $\left(P\left(x^{\prime}, x^{\prime \prime}\right) P^{*}\left(x^{\prime \prime}, x^{\prime \prime \prime}\right) h, h\right) \geqslant \frac{1}{M}\|h\|^{2} \quad 0<M<+\infty$;

б) $\left\|P\left(x^{\prime}, x^{\prime \prime}\right) h\right\| \geqslant \frac{1}{\sqrt{M}}\|h\|$;

в) $\left\|P\left(x^{\prime}, x^{\prime \prime}\right)\right\| \leqslant \sqrt{K}$;

$3^{\circ} \quad M K<2$.

Тогда уравнение (1) имеет в сфере (22) единственное решение $x^{*}$, к.которому последовательность (20) сходится со скоростью

$$
\left\|x^{*}-x_{n}\right\| \leqslant \frac{\sqrt{M} \eta_{0}}{1-q} g^{n} \quad n=0,1,
$$

że

$$
q=\sqrt{2-\frac{2}{M K}}
$$

Доказ ательство: Аналогично доказательству теоремы 1, получим:

$$
\begin{aligned}
& \left\|P\left(x_{n+1}\right)\right\|^{2}=\left\|P\left(x_{n}\right)\right\|^{2}- \\
& \quad-2 \frac{\left\|P^{*}\left(x_{n}, x_{n-1}\right) P\left(x_{n}\right)\right\|^{2}}{\left\|P\left(x_{n}, x_{n-1}\right) P^{*}\left(x_{n}, x_{n-1}\right) P\left(x_{n}\right)\right\|^{2}}\left(P\left(x_{n+1}, x_{n}\right) P^{*}\left(x_{n}, x_{n-1}\right) P\left(x_{n}\right), P\left(x_{n}\right)\right)+ \\
& +\frac{\left\|P^{*}\left(x_{n}, x_{n-1}\right) P\left(x_{n}\right)\right\|^{4}}{\left\|P\left(x_{n}, x_{n-1}\right) P^{*}\left(x_{n}, x_{n-1}\right) P\left(x_{n}\right)\right\|^{4}}\left\|P\left(x_{n+1}, x_{n}\right) P^{*}\left(x_{n}, x_{n-1}\right) P\left(x_{n}\right)\right\|^{2}
\end{aligned}
$$

Поскольку

$$
\begin{array}{r}
\frac{\left\|P^{*}\left(x_{n}, x_{n-1}\right) P\left(x_{n}\right)\right\|^{4}}{\left\|P\left(x_{n}, x_{n-1}\right) P^{*}\left(x_{n}, x_{n-1}\right) P\left(x_{n}\right)\right\|^{4}}=\frac{\left(P\left(x_{n}, x_{n-1}\right) P^{*}\left(x_{n}, x_{n-1}\right) P\left(x_{n}\right), P\left(x_{n}\right)\right)^{2}}{\left\|P\left(x_{n}, x_{n-1}\right) P^{*}\left(x_{n}, x_{n-1}\right) P\left(x_{n}\right)\right\|^{4}} \leqslant \\
\leqslant \frac{\left\|P\left(x_{n}, x_{n-1}\right) P^{*}\left(x_{n}, x_{n-1}\right) P\left(x_{n}\right)\right\|^{2}\left\|P\left(x_{n}\right)\right\|^{2}}{\left\|P\left(x_{n}, x_{n-1}\right) P^{*}\left(x_{n}, x_{n-1}\right) P\left(x_{n}\right)\right\|^{4}}=\frac{\left\|P\left(x_{n}\right)\right\|^{2}}{\left\|P\left(x_{n}, x_{n-1}\right) P^{*}\left(x_{n}, x_{n-1}\right) P\left(x_{n}\right)\right\|^{2}},
\end{array}
$$

то на основании формулы (25) имеем:

$$
\begin{gathered}
\left\|P\left(x_{n+1}\right)\right\|^{2} \leqslant\left\|P\left(x_{n}\right)\right\|^{2}-\frac{\left\|P^{*}\left(x_{n}, x_{n-1}\right) P\left(x_{n}\right)\right\|^{2}}{\left\|P\left(x_{n}, x_{n-1}\right) P^{*}\left(x_{n}, x_{n-1}\right) P\left(x_{n}\right)\right\|^{2}} \times \\
X\left[2\left(P\left(x_{n+1}, x_{n}\right) P^{*}\left(x_{n}, x_{n-1}\right) P\left(x_{n}\right), P\left(x_{n}\right)\right)-\left\|P\left(x_{n+1}, x_{n}\right)\right\|^{2}\left\|P\left(x_{n}\right)\right\|^{2}\right] \leqslant \\
\leqslant\left[1-\frac{\left\|P^{*}\left(x_{n}, x_{n-1}\right) P\left(x_{n}\right)\right\|^{2}}{K\left\|P^{*}\left(x_{n}, x_{n-1}\right) P\left(x_{n}\right)\right\|^{2}}\left(\frac{2}{M}-K\right)\right]\left\|P\left(x_{n}\right)\right\|^{2} \leqslant \\
\leqslant\left(2-\frac{2}{M K}\right)\left\|P\left(x_{n}\right)\right\|^{2}=q^{2}\left\|P\left(x_{n}\right)\right\|^{2}<\left\|P\left(x_{n}\right)\right\|^{2}
\end{gathered}
$$


откуда, как и при доказательстве теоремы 1, вытекают утверждения нашей теоремы. Отметим только, что в данном случае при $n=0,1, \ldots$

$$
\text { i| } x_{n+1}-x_{n}\|\leqslant \sqrt{M}\| P\left(x_{n}\right) \| \text {. }
$$

5. Аналогично можно доказать и теорему о сходимости интерполяционного аналога итерационного метода с минимальными ошибками

$$
x_{n+1}=x_{n}-\frac{\left\|P\left(x_{n}\right)\right\|^{2}}{\left\|P^{*}\left(x_{n}, x_{n-1}\right) P\left(x_{n}\right)\right\|^{2}} P^{*}\left(x_{n}, x_{n-1}\right) P\left(x_{n}\right) \text {, }
$$

т. е. в классе методов (3) выбрано

$$
\varepsilon_{n}=\frac{\left\|P\left(x_{n}\right)\right\|^{2}}{\left\|P^{*}\left(x_{n}, x_{n-1}\right) P\left(x_{n}\right)\right\|^{2}} .
$$

Т е орем а 4: Пусть выполнены условия:

$1^{\circ} \quad\left\|P\left(x_{0}\right)\right\| \leqslant \eta_{0} ; \quad\left\|x_{0}-x_{-1}\right\| \leqslant \gamma ; \quad x_{0} \neq x_{-1} ;$

$2^{\circ}$ длякаждого $x^{\prime}, x^{\prime \prime}, x^{\prime \prime \prime} \in S\left[x_{0} ; \max \left(\gamma ; \frac{\sqrt{M} \eta_{0}}{1-q}\right)\right]$

“

a) $\left(P\left(x^{\prime}, x^{\prime \prime}\right) P^{*}\left(x^{\prime \prime}, x^{\prime \prime \prime}\right) h, h\right) \geqslant \frac{1}{M}\|h\|^{2} \quad 0<M<+\infty$;

б) $\left\|P\left(x^{\prime}, x^{\prime \prime}\right)\right\| \leqslant \sqrt{K}$;

$3^{\circ} \quad M K<2$.

Тогда уравнение (1) имеет в сфере (31) единственное решение $x^{*}$, к которому последовательность (29) сходится со скоростью

$$
\left\|x^{*}-x_{n}\right\| \leqslant \frac{\sqrt{M} \eta_{0}}{1-q} q^{n} \quad n=0,1, \ldots,
$$

гдe

$$
q=\sqrt{2-\frac{2}{M K}}
$$

6. Если известно, что оператор $P\left(x^{\prime}, x^{\prime \prime}\right)$ удовлетворяет в некоторой сфере условию Липшица, можно доказать теорему о сходимости методов класса (3). Иллюстрируем это на примере метода (20).

Т е о рем а 5: Пусть выполнены условия:

$1^{\circ} \quad\left\|P\left(x_{0}\right)\right\| \leqslant \eta_{0} ;\left\|x_{0}-x_{-1}\right\| \leqslant \sqrt{M} \gamma ; \quad x_{0} \neq x_{-1} ;$

$2^{\circ}$ для каждого $x^{\prime}, x^{\prime \prime}, x^{\prime \prime \prime} \in S\left[x_{0} ; \max \left(\sqrt{M} \gamma ; \frac{\sqrt{M}}{1-q} \eta_{0}\right)\right]$

u hєH справедливы оценки:

a) $\left\|P\left(x^{\prime}, x^{\prime \prime}\right) h\right\| \geqslant \frac{1}{\sqrt{M}}\|h\| ;\left\|P^{*}\left(x^{\prime}, x^{\prime \prime}\right) h\right\| \geqslant \frac{1}{\sqrt{M}}\|h\| ; \quad 0<M<+\infty$; 
б) $\left\|P\left(x^{\prime}, x^{\prime \prime}\right)\right\| \leqslant \sqrt{K}$;

в) $\left\|P\left(x^{\prime}, x^{\prime \prime}\right)-P\left(x^{\prime \prime}, x^{\prime \prime \prime}\right)\right\| \leqslant L\left\|x^{\prime}-x^{\prime \prime \prime}\right\|$;

$3^{\circ}(M K+1) M L\left(\eta_{0}+\delta\right)<2$, где $\delta=\max \left(\eta_{0} ; \gamma\right)$.

Тогда уравнение (1) имеет в сфере (34) единственное решение $x^{*}$, к которому сходится последовательность (20). Справедливы оценки:

$$
\left\|x^{*}-x_{n}\right\| \leqslant \frac{\sqrt{M} \eta_{0}}{1-q} q^{n} \quad n=0,1, \ldots,
$$

.əेe

$$
q=\frac{M K-1}{M K+1}+M L\left(\eta_{0}+\delta\right)
$$

Доказ а тельство: Так как в данном случае

$$
\begin{gathered}
\left\|P\left(x_{n}\right)+P\left(x_{n}, x_{n-1}\right)\left(x_{n+1}-x_{n}\right)\right\|^{2}= \\
=\left\|P\left(x_{n}\right)\right\|^{2}-\frac{\left(P\left(x_{n}, x_{n-1}\right) P^{*}\left(x_{n}, x_{n-1}\right) P\left(x_{n}\right), P\left(x_{n}\right)\right)^{2}}{\left\|P\left(x_{n}, x_{n-1}\right) P^{*}\left(x_{n}, x_{n-1}\right) P\left(x_{n}\right)\right\|^{2}}
\end{gathered}
$$

и оператор $P\left(x_{n}, x_{n-1}\right) P^{*}\left(x_{n}, x_{n-1}\right)$ является самосопряженным, то, используя неравенство Грейба-Рейнбольдта $\left[{ }^{8}\right]$, получим:

$\left\|P\left(x_{n}\right)+P\left(x_{n}, x_{n-1}\right)\left(x_{n+1}-x_{n}\right)\right\| \leqslant \frac{M K-1}{M K+1}\left\|P\left(x_{n}\right)\right\| \quad n=0,1, \ldots$

На основании аналога интерполяционной формулы Ньютона [5]

$$
\begin{gathered}
\left\|P\left(x_{n+1}\right) ! \leqslant\right\| P\left(x_{n}\right)+P\left(x_{n}, x_{n-1}\right)\left(x_{n+1}-x_{n}\right)\|+\|\left[P\left(x_{n+1}, x_{n}\right)-\right. \\
\left.-P\left(x_{n}, x_{n-1}\right)\right]\left(x_{n+1}-x_{n}\right) \| .
\end{gathered}
$$

Используя неравенства (28) и (38), получим здесь при $n=0$ $\left\|P\left(x_{1}\right)\right\| \leqslant \frac{M K-1}{M K+1}\left\|P\left(x_{0}\right)\right\|+L\left\|x_{1}-x_{-1}\right\|\left\|x_{1}-x_{0}\right\| \leqslant$

$$
\begin{aligned}
& \leqslant \frac{M K-1}{M K+1}\left\|P\left(x_{0}\right)\right\|+L\left(\left\|x_{1}-x_{0}\right\|^{2}+\left\|x_{1}-x_{0}\right\|\left\|x_{0}-x_{-1}\right\|\right) \leqslant \\
& \leqslant\left[\frac{M K-1}{M K+1}+M L\left(\eta_{0}+\gamma\right)\right]\left\|P\left(x_{0}\right)\right\| \leqslant q\left\|P\left(x_{0}\right)\right\|
\end{aligned}
$$

и при $n>1$ по индукции

$\left\|P\left(x_{n+1}\right)\right\| \leqslant \frac{M K-1}{M K+1}\left\|P\left(x_{n}\right)\right\|+L\left\|x_{n+1}-x_{n-1}\right\|\left\|x_{n+1}-x_{n}\right\| \leqslant$

$$
\begin{aligned}
& \leqslant \frac{M K-1}{M K+1}\left\|P\left(x_{n}\right)\right\|+L\left(\left\|x_{n+1}-x_{n}\right\|^{2}+\left\|x_{n+1}-x_{n}\right\|\left\|x_{n}-x_{n-1}\right\|\right) \leqslant \\
& \leqslant\left[\frac{M K-1}{M K+1}+M L\left(\left\|P\left(x_{n}\right)\right\|+\left\|P\left(x_{n-1}\right)\right\|\right]\left\|P\left(x_{n}\right)\right\| \leqslant\right. \\
& \leqslant\left[\frac{M K-1}{M K+1}+M L q^{n-1}(1+q) \eta_{0}\right]\left\|P\left(x_{n}\right)\right\|<q\left\|P\left(x_{n}\right)\right\|
\end{aligned}
$$

откуда вытекают утверждения теоремы. Единственность решения в сфеpe (34) вытекает из условия $2 \mathrm{a}$. 
3 а мечание 2: Так как в теоремах 3 и 5 требуется выполнение условия $\left\|P\left(x^{\prime}, x^{\prime \prime}\right) h\right\| \geqslant \frac{1}{\sqrt{M}}\|h\|$, то имеет место следующая апостериорная оценка погрешности (ср. [2])

$$
\left\|x^{*}-x_{n}\right\| \leqslant \sqrt{M}\left\|P\left(x_{n}\right)\right\| \quad n=0,1, \ldots .
$$

Действительно, из соотношений

$$
\left\|P\left(x_{n}\right)\right\|^{2}=\left\|P\left(x^{*}\right)-P\left(x_{n}\right)\right\|^{2}=\left\|P\left(x^{*}, x_{n}\right)\left(x^{*}-x_{n}\right)\right\|^{2} \geqslant \frac{1}{M}\left\|x^{*}-x_{n}\right\|^{2}
$$

вытекает (41).

7. Чтобы иллюстрировать практическое применение методов класса (3), рассмотрим нелинейную систему двух уравнений

$$
\left\{\begin{array}{l}
f(u, v)=0 \\
g(u, v)=0
\end{array}\right.
$$

В данном случае $H=R^{2}, x=(u, v), P(x)=(f, g),\|x\|=\sqrt{u^{2}+v^{2}}$.

\section{Определяя}

имеем:

$$
P\left(x^{\prime}, x^{\prime \prime}\right)=\left(\begin{array}{cc}
\frac{f\left(u^{\prime}, v^{\prime}\right)-f\left(u^{\prime \prime}, v^{\prime}\right)}{u^{\prime}-u^{\prime \prime}} & \frac{f\left(u^{\prime \prime}, v^{\prime}\right)-f\left(u^{\prime \prime}, v^{\prime \prime}\right)}{v^{\prime}-v^{\prime \prime}} \\
\frac{g\left(u^{\prime}, v^{\prime}\right)-g\left(u^{\prime \prime}, v^{\prime}\right)}{u^{\prime}-u^{\prime \prime}} & \frac{g\left(u^{\prime \prime}, v^{\prime}\right)-g\left(u^{\prime \prime}, v^{\prime \prime}\right)}{v^{\prime}-v^{\prime \prime}}
\end{array}\right),
$$

$$
P^{*}\left(x^{\prime}, x^{\prime \prime}\right)=\left(\begin{array}{ll}
\frac{f\left(u^{\prime}, v^{\prime}\right)-f\left(u^{\prime \prime}, v^{\prime}\right)}{u^{\prime}--u^{\prime \prime}} & \frac{g\left(u^{\prime}, v^{\prime}\right)-g\left(u^{\prime \prime}, v^{\prime}\right)}{u^{\prime}-u^{\prime \prime}} \\
\frac{f\left(u^{\prime \prime}, v^{\prime}\right)-f\left(u^{\prime \prime}, v^{\prime \prime}\right)}{v^{\prime}-v^{\prime \prime}} & \frac{g\left(u^{\prime \prime}, v^{\prime}\right)-g\left(u^{\prime \prime}, v^{\prime \prime}\right)}{v^{\prime}-v^{\prime \prime}}
\end{array}\right) \text {. }
$$

Для решения системы (33) методы (3) принимают вид

$$
\begin{aligned}
u_{n+1} & =u_{n}-\varepsilon_{n}\left[\frac{f\left(u_{n}, v_{n}\right)-f\left(u_{n-1}, v_{n}\right)}{u_{n}-u_{n-1}} f\left(u_{n}, v_{n}\right)+\frac{g\left(u_{n}, v_{n}\right)-g\left(u_{n-1}, v_{n}\right)}{u_{n}-u_{n-1}} g\left(u_{n}, v_{n}\right)\right] \\
v_{n+1}^{\prime} & =v_{n}-\varepsilon_{n}\left[\frac{f\left(u_{n-1}, v_{n}\right)-f\left(u_{n-1}, v_{n-1}\right)}{v_{n}-v_{n-1}} f\left(u_{n}, v_{n}\right)+\right. \\
& \left.+\frac{g\left(u_{n-1}, v_{n}\right)-g\left(u_{n-1}, v_{n-1}\right)}{v_{n}-v_{n-1}} g\left(u_{n}, v_{n}\right)\right] \quad n=0,1, \ldots
\end{aligned}
$$

Исходя из теорем, изложенных в пунктах 2 и 3 статьи, легко сформулировать условия сходимости последовательностей (46) к решению $x^{*}=\left(u^{*}, v^{*}\right)$ системы (43).

\section{Л И Т Е Р А Т Р А}

1. М. М. В а йн бе рг, О сходимости процесса наискорейшего спуска для нелинейных уравнений, Сибирский матем. журнал, 2, 2, 1961.

2. Л. А. К и в стик, Об одной модификации итерационного метода с минимальными невязкими для решения нелинейных операторных уравнений, ДАН, 136, 1,1961 . 
3. М. А. К р асн ос ельски й и С. Г. К рейн, Итерационный процесс с минимальными невязками, Матем. сборник, 31, (73): 2, 1952.

4. Е. И. Линьков, О методе наискорейшего спуска для нелинейных уравнений, Ученые записки Московск. обл. пед. ин-та, 96, вып. 6, 1960.

5. А. С. С ергеев, О методе хорд, Сибирский матем. журнал, 2, 2, 1961.

6. С. У льм, Об интерполяционных методах решения нелинейных уравнений в пространстве Банаха, Изв. АН ЭССР. Сер. физ.-матем. и техн. наук, 12, 1, 1963.

7. В. М. Фр и д м а н, Итеративный процесс с минимальными ошибками для нелинейного операторного уравнения, ДАН, 139, 5, 1961.

8. W. Greub, W. Rheinboldt, On a generalization of an inequality of L. V. Kantorovich, Proc. Am. Math. Soc., 10, 3, 1959.

Институт кибернетики

Академии наук Эстонской ССР
Поступила в редакцию

4. X 1962

\title{
UHEST ITERATSIOONMENETLUSTE KLASSIST HILBERTI RUUMIS
}

\author{
S. Ulm, \\ füüsika-matemaatikateaduste kandidaat
}

Resümee

Reaalses Hilberti ruumis vaadeldakse mittelineaarse operaatorvõrrandi $P(x)=0$ lahendamiseks iteratsioonmenetluste klassi

$$
x_{n+1}=x_{n}-\varepsilon_{n} P^{*}\left(x_{n}, x_{n-1}\right) P\left(x_{n}\right) \quad(n=0,1, \ldots),
$$

kus $\varepsilon_{n}$ on positiivsed arvud, $P\left(x^{\prime}, x^{\prime \prime}\right)$ - operaatori $P$ esimest järku diferentssuhte analoog ja $P^{*}\left(x^{\prime}, x^{\prime \prime}\right)$ - lineaarse operaatori $P\left(x^{\prime}, x^{\prime \prime}\right)$ kaasoperaator. Vaadeldakse kaht jada $\left\{\varepsilon_{n}\right\}$ valiku juhtu ja meetodite rakendamist mittelineaarsetele võrrandisüsteemidele.
Eesti NSV Teaduste Akadeemia
Küberneetika Instituut
Saabus toimetusse
4. X 1962

\section{UBER EINE KLASSE VON ITERATIONSVERFAHREN IM HILBERTSCHEN RAUM}

\section{S. Uim}

\section{Zusanımenfassung}

Für die angenäherte Lösung der Operatorgleichung $P(x)=0$ im Hilbertraum wird die Klasse der Iterationsverfahren

$$
x_{n+1}=x_{n}-\varepsilon_{n} P^{*}\left(x_{n}, x_{n-1}\right) P\left(x_{n}\right) \quad(n=0,1, \ldots)
$$

untersucht, wobei $\varepsilon_{n}$ positive Zahlen sind, $P\left(x^{\prime}, x^{\prime \prime}\right)$ - das Analogon des Differenzenquotients erster Ordnung für den Operator $P$ und $P^{*}\left(x^{\prime}, x^{\prime \prime}\right)$ - der konjugierte Operator für den linearen Operator $P\left(x^{\prime}, x^{\prime \prime}\right)$. Es werden zwei Spezialfälle der Folge $\left\{\varepsilon_{n}\right\}$ und die Anwendung der Verfahren auf nichtlineare Gleichungssysteme behandelt.

\section{Institut für Kybernetik}

der Akademie der Wissenschaften der Estnischen SSR
Eingegangen am 4. Okt. 1962 